\title{
WEAK COMPACTNESS IN THE SPACE OF VECTOR MEASURES ${ }^{1}$
}

\author{
BY JAMES K. BROOKS
}

Communicated by B. Yood, July 26, 1971

Abstract. A necessary and sufficient condition for weak compactness in the space of vector measures is given.

1. Introduction and statement of the theorem. Weak compactness in the space of set functions has been studied by a number of authors. Dubrovskii [6], Grothendieck [9], Bartle, Dunford and Schwartz [1] obtained necessary and sufficient conditions for weak compactness in the space of countably additive scalar measures. Leader [10] and Porcelli [13] treated the finitely additive scalar case. Chatterji [4] considered weak compactness in $L_{1}(\mathfrak{X})$; a slightly more general case was studied by Batt and Berg [2].

In this paper we give necessary and sufficient conditions for weak compactness in a very general setting, which includes the above results as special cases. Details and applications will be presented elsewhere. Our theorem is the following (definitions are given in the next section).

THEOREM. Let $\mathfrak{X}$ be a reflexive Banach space and let $\mathscr{R}$ be a ring of sets. $A$ set $K \subset f a(\mathscr{R}, \mathfrak{X})$ is conditionally weakly compact if and only if $K$ is bounded and the set of measures $\{v(\mu): \mu \in K\}$ is uniformly additive.

REMARK 1 . In the case $K$ is weakly compact, there exists a positive bounded finitely additive set function $\lambda$ such that $\{v(\mu): \mu \in K\}$ is uniformly absolutely continuous with respect to $\lambda$. Conversely, if $K$ is bounded and such a $\lambda$ exists, then $K$ is conditionally weakly compact. The hypothesis of reflexivity in the necessary part of Theorem 1 can be omitted.

COROLlaRY 1 . The above theorem remains valid if $f a(\mathscr{R}, \mathfrak{X})$ is replaced by $c a(\mathscr{R}, \mathfrak{X})$.

REMARK 2. The theorem is false for every $\mathfrak{X}$ which is not reflexive. This observation was made by J. J. Uhl, Jr. In fact, if $\mathfrak{X}$ is not reflexive, choose a sequence $\left\{x_{n}\right\}$ in the unit sphere of $\mathfrak{X}$ which has no weakly convergent subsequences. Assume $(S, \Sigma, \mu)$ is a measure space; let $E$ be a measurable set of positive measure. The subspace $\left\{x \zeta_{E}: x \in X\right\}$ of $L_{1}(\mu, \mathfrak{X})$ is linearly homeomorphic to $\mathfrak{X}$. Note that the set $K=\left\{x_{n} \xi_{E}: n=1,2, \ldots\right\}$ is not conditionally weakly compact.

AMS 1970 subject classifications. Primary 46G10; Secondary 28A55.

Key words and phrases. Stone algebra, weak compactness, Radon-Nikodym-Phillips theorem, Bartle-Dunford-Schwartz theorem.

${ }^{1}$ This work was supported by the National Science Foundation Grant GP-28617. 
COROllary 2. $f a(\mathscr{R}, X)$ is weakly sequentially complete if $X$ is reflexive.

2. Notation and definition. $\mathfrak{X}$ denotes a Banach space with norm \|| and conjugate space $\mathfrak{X}^{*}$. Let $\mathscr{R}$ be a ring of subsets of a set $S$. For a set function $\mu: \mathscr{R} \rightarrow \mathfrak{X}$, define the total variation set function on the power set of $S$ by $v(\mu)(A)=\sup \sum\left\|\mu\left(R_{i}\right)\right\|$, where the supremum is taken over all finite disjoint families of sets $R_{i}$ contained in $A$. $f a(\mathscr{R}, \mathfrak{X})$ is the Banach space of all finitely additive set functions $\mu$ defined on $\mathscr{R}$ with $v(\mu)(S)<\infty$. The norm of $\mu$ is $v(\mu)(S) . c a(\mathscr{R}, \mathfrak{X})$ is the subspace of $f a(\mathscr{R}, \mathfrak{X})$ consisting of countably additive set functions. $\sigma(\Sigma)$ denotes the $\sigma$-algebra of sets generated by $\Sigma . L_{1}(S, \mathscr{A}, v, \mathfrak{X})$ is the space of Bochner integrable functions relative to the measure space $(S, \mathscr{A}, v) ; L_{\infty}(S, \mathscr{A}, v, \mathfrak{X})$ denotes the class of $\mathscr{A}$-measurable essentially bounded $\mathfrak{X}$-valued functions. A family $\Gamma$ of finitely additive scalar measures of bounded variation defined on $\mathscr{R}$ is uniformly additive if for every disjoint sequence of sets $R_{i}, \lim _{n} \sum_{i=n}^{\infty}\left|\mu\left(R_{i}\right)\right|$ $=0$ uniformly for $\mu \in \Gamma$. Weak convergence is denoted by $\stackrel{m}{\rightarrow}$.

3. Brief outline of the proof. I. Assume $\mathscr{R}=\Sigma$ is a $\sigma$-algebra. Let $K=\left\{\mu_{i}\right\}_{i=1}^{\infty} \subset c a(\Sigma, \mathfrak{X})$. Suppose $\mu_{0}: \Sigma \rightarrow \mathfrak{X}$ is a set function such that $\mu_{i}(E) \stackrel{w}{\rightarrow} \mu_{0}(E)$ in $\mathfrak{X}$ for every $E \in \Sigma$. Then for every $x^{*} \in \mathfrak{X}^{*}, x^{*} \mu_{0}=\lim x^{*} \mu_{i}$. By the Nikodym theorem [7, p. 160], $x^{*} \mu_{0}$ is countably additive, and the Orlicz-Pettis theorem [11] implies that $\mu_{0}$ is countably additive on $\Sigma$. The expression $\sum_{i=1}^{n}\left\|\mu_{0}\left(E_{i}\right)\right\|=\lim _{k} \sum_{i=1}^{n} x_{i}^{*} \mu_{k}\left(E_{i}\right)$ for appropriate $x_{i}^{*} \in \mathfrak{X}^{*}$, $\left\|x_{i}^{*}\right\| \leqq 1$, shows that $v\left(\mu_{0}\right)(S) \leqq \underline{\lim } v\left(\mu_{i}\right)(S)$; hence $\mu_{0} \in c a(\Sigma, \mathfrak{X})$. Let $3_{0}$ be the rational span of $\left\{\mu_{i}\right\}_{i=0}^{\infty}$. Obtain a countable algebra $\mathscr{A}_{0} \subset \Sigma$ such that $v\left(\beta / \mathscr{A}_{0}\right)(S)=V(\beta)(S), \beta \in 3_{0}$. We then show that $\beta \in 3$ implies:

$$
v(\beta / \mathscr{A})(S)=v(\beta)(S),
$$

where 3 is the closure in $c a(\Sigma, \mathfrak{X})$ of $\mathfrak{Z}_{0}$ and $\mathscr{A}=\sigma\left(\mathscr{A}_{0}\right)$. Let the separable space $\mathfrak{X}_{0}$ be the closure of $\operatorname{span}\left\{\beta(A): A \in \mathscr{A}_{0}, \beta \in \mathfrak{Z}_{0}\right\}$. Using the HahnBanach theorem and the Hahn extension theorem for measures, we obtain: $\beta(A) \in \mathfrak{X}_{0}$ whenever $\beta \in \mathcal{Z}$ and $A \in \mathscr{A}$. This fact and (*) yield the isometry $\pi: 3 \rightarrow c a\left(\mathscr{A}, \mathfrak{X}_{0}\right)$, where $\pi(\beta)=\beta / \mathscr{A}, \beta \in 3$. Let $v=\sum_{i=0}^{\infty} 2^{-i} v\left(\mu_{i}\right)$. Since $\mathfrak{X}_{0}$ is reflexive, by the Radon-Nikodym-Phillips theorem [12], we obtain functions $f_{i}$ in $L_{1}\left(S, \mathscr{A}, v, \mathfrak{X}_{0}\right) \equiv L_{1}\left(\mathfrak{X}_{0}\right)$ such that $f_{i}=d \mu_{i} / d v, i=0,1, \ldots$. Since $\mathfrak{X}_{0}$ is reflexive, $\mathfrak{X}_{0}^{*}$ is separable, and thus $L_{1}\left(\mathfrak{X}_{0}\right)^{*}=L_{\infty}\left(S, \mathscr{A}, v, \mathfrak{X}_{0}^{*}\right)$ $\equiv L_{\infty}\left(\mathfrak{X}_{0}^{*}\right)$ (cf. Dinculeanu [5, p. 282]). $\mu_{i}(E) \stackrel{w}{\rightarrow} \mu_{0}(E)$ for every $E$ implies that $\int h f_{i} d v \rightarrow \int h f_{0} d v$ for simple functions $h \in L_{\infty}\left(\mathfrak{X}_{0}^{*}\right)$. Since the $v\left(\mu_{i}\right)$ are uniformly additive and absolutely continuous with respect to $v$, a result of Gould [8, p. 199] implies that the $v\left(\mu_{i}\right)$ are uniformly absolutely continuous with respect to $v$. Using this fact together with Egoroff's theorem for $\mathfrak{X}_{0}^{*}$-valued measurable functions and the boundedness of $\left\{v\left(\mu_{i}\right)(S)\right\}$, one can show that $\int h f_{i} d v \rightarrow \int h f_{0} d v, h \in L_{\infty}\left(\mathfrak{X}_{0}^{*}\right)$; this in turn implies that $\mu_{i} \stackrel{w}{\rightarrow} \mu_{0}$ in $c a(\Sigma, \mathfrak{X})$. 
II. Suppose $K \subset c a(\Sigma, \mathfrak{X})$, where $\Sigma$ is a $\sigma$-algebra. By the EberleinŠmulian theorem, it suffices to consider a sequence $\left\{\mu_{i}\right\} \subseteq K$. As in I, consider $\left\{\mu_{i}\right\} \subset c a\left(\mathscr{A}, \mathfrak{X}_{0}\right), \mathfrak{X}_{0}$ separable. An application of the Bartle-DunfordSchwartz weak compactness theorem $[1]$ shows that $\left\{x^{*} \mu_{i}\right\}$ is conditionally weakly compact for every $x^{*} \in \mathfrak{X}^{*}$. Let $x_{n}^{*}$ be dense in the unit sphere of $\mathfrak{X}_{0}^{*}$. By a diagonal process obtain $\left\{\mu_{i_{k}}\right\}$ such that $\lim _{k} x_{n}^{*} \mu_{i_{k}}(E)$ exists for every $E \in \mathscr{A}$ and every $n$. The limit also exists then for every $x^{*} \in \mathfrak{X}_{0}^{*}$. Define $\mu(E): \mathfrak{X}_{0}^{*} \rightarrow$ (scalar field) by $\mu(E) x^{*}=\lim x^{*} \mu_{i_{k}}(E)$. Then $\mu(E) \in \mathfrak{X}_{0}^{* *}=\mathfrak{X}_{0}$ and $\mu_{i_{k}}(E) \stackrel{w}{\rightarrow} \mu(E), E \in \mathscr{A}$. As in I, $\mu_{i_{k}} \stackrel{w}{\rightarrow} \mu$ in $c a\left(\mathscr{A}, \mathfrak{X}_{0}\right)$. Since closed subspaces contain weak limits of elements in the subspace, $\mu_{i_{k}} \stackrel{w}{\rightarrow} \pi^{-1}(\mu)$ in $c a(\Sigma, \mathfrak{X})$.

III. Let $K \subset f a(\Sigma, \mathfrak{X})$, where $\Sigma$ is an algebra. Let $\Sigma_{1}$ be the Stone algebra [7, p. 312] of all open-closed subsets of the compact Hausdorff space $S_{1} ; \Sigma_{2}=\sigma\left(\Sigma_{1}\right)$. For a scalar or vector finitely additive set function $\mu$ of bounded variation defined on $\Sigma$, let $\tau(\mu)$ be its extension on $\Sigma_{2} \cdot \tau: f a(\Sigma, \mathfrak{X})$ $\rightarrow c a\left(\Sigma_{2}, \mathfrak{X}\right)$ is an isometry (cf. Uhl [14]). It can be proved that on $\Sigma_{2}$, $v(\tau \mu) \leqq \tau(v(\mu))$. The conditional weak compactness of $K$ is established by showing the uniform additivity of $\tau(K)$. This is indicated in the next step.

IV. Let $\Gamma$ be a bounded set of scalar countably additive set functions defined on an algebra $\Sigma_{0}$. If $\Gamma$ is a uniformly additive family, then the extensions of $\Gamma$ to $\sigma\left(\Sigma_{0}\right)$ are also uniformly additive. The technique used to prove this is similar to that found in Brooks [3]. A diagonal process is used in addition to results of Leader [10] and Porcelli [13].

V. Let $\mathscr{R}$ be a ring $(S \notin \mathscr{R}), \Sigma$ the algebra generated by $\mathscr{R} . \Sigma$ consists of sets of the form $R$ or $R \cup\left(S-R_{0}\right), R, R_{0} \in \mathscr{R}\left(R \cap\left(S-R_{0}\right)=\varnothing\right)$. For $\mu \in f a(\mathscr{R}, \mathfrak{X})$, define $\tilde{\mu}$ on $\Sigma$ by $\tilde{\mu}\left(R \cup\left(S-R_{0}\right)\right)=\mu(R)-\mu\left(R_{0}\right)$. Thus $v(\mu)(S) \leqq v(\tilde{\mu})(S) \leqq 2 v(\mu)(S) . K$ is uniformly additive on $\mathscr{R}$ if and only if $K$ is uniformly additive on $\Sigma$. Using the maps $\beta \rightarrow \beta / \mathscr{R}(\beta \in f a(\Sigma, \mathfrak{X}))$ and $\beta \rightarrow \tilde{\beta}(\beta \in f a(\mathscr{R}, \mathfrak{X}))$, we reduce $\mathrm{V}$ to III.

VI. The converse (without reflexivity) is proved by sliding hump methods in sequence spaces.

VII. Remark 1 follows from the result: If a family $\Gamma$ of finitely additive scalar measures is bounded, then $\Gamma$ is uniformly additive if and only if there exists a bounded positive finitely additive measure $\lambda$ such that $\Gamma$ is uniformly absolutely continuous with respect to $\lambda$ and $\lambda(E) \leqq \sup \{v(\mu)(E): \mu \in \Gamma\}$. Results from [1], [3] and [13] are used to prove this.

Corollary 1 follows from the theorem by observing that $c a(\mathscr{R}, \mathfrak{X})$ is a closed subspace of $f a(\mathscr{R}, \mathfrak{X})$ and therefore contains weak limits in $f a(\mathscr{R}, \mathfrak{X})$ of elements belonging to $c a(\mathscr{R}, \mathfrak{X})$.

REMARK 3. The author and N. Dinculeanu have recently extended the above theorem to the space of vector measures of local finite variation. A "Synthesis theorem" concerning the existence of a control measure for a 
family of locally equivalent measures is used to show the existence of a positive measure $\lambda$ such that weakly compact sets in this locally convex linear topological space are locally uniformly absolutely continuous with respect to it.

REMARK 4. The author has obtained criteria for strong compactness in the space of vector measures with local finite variation. Details will appear elsewhere.

\section{REFERENCES}

1. R. G. Bartle, N. Dunford and J. Schwartz, Weak compactness and vector measures, Canad. J. Math. 7 (1955), 289-305. MR 16, 1123.

2. J. Batt and E. J. Berg, Linear bounded transformations on the space of continuous functions, J. Functional Analysis 4 (1969), 215-239. MR 40 \# 1798.

3. J. K. Brooks, On the existence of a control measure for strongly bounded vector measures, Bull. Amer. Math. Soc. 77 (1971), 999-1001.

4. S. D. Chatterji, Weak convergence in certain special Banach spaces, MRC Technical Sum. Report \# 443, University of Wisconsin, Madison, Wis., 1963.

5. N. Dinculeanu, Vector measures, Internat. Series of Monographs in Pure and Appl. Math., vol. 95, Pergamon Press, Oxford; VEB Deutscher Verlag, Berlin, 1967. MR 34 \# 6011.

6. V. M. Dubrovskiǐ, On the basis of a family of completely additive functions of sets and on the properties of uniform additivity and equi-continuity, Dokl. Akad. Nauk SSSR 58 (1947), 737-740. (Russian) MR 9, 275.

7. N. Dunford and J. T. Schwartz, Linear operators. I: General theory, Interscience, New York, 1958. MR 22 \#8302.

8. G. G. Gould, Integration over vector-valued measures, Proc. London Math. Soc. (3) 15 (1965), 193-225. MR 30 \# 4894.

9. A. Grothendieck, Sur les applications linéaires faiblement compactes d'espaces du type $C(K)$, Canad. J. Math. 5 (1953), 129-173. MR 15, 438.

10. S. Leader, The theory of $L^{p}$-spaces for finitely additive set functions, Ann. of Math. (2) 58 (1953), 528-543. MR 15, 326.

11. B. J. Pettis, On integration in vector spaces, Trans. Amer. Math. Soc. 44 (1938), $277-304$.

12. R. S. Phillips, On weakly compact subsets of a Banach space, Amer. J. Math. 65(1943), 108-136. MR 4, 218.

13. P. Porcelli, Two embedding theorems with applications to weak convergence and compactness in spaces of additive type functions, J. Math. Mech.9(1960), 273-292. MR 23 \# A2034.

14. J. J. Uhl, Jr., Orlicz spaces of finitely additive set functions, Studia Math. 29 (1967), 19-58. MR 37 \# 1985.

Department of Mathematics, University of Florida, Gainesville, Florida 32601 